\title{
Anesthetic management of super-elderly patients with remimazolam: a report of two cases
}

\author{
Junko Nakayama*, Tomomi Ogihara' ${ }^{1}$, Rui Yajima², Yasushi Innami and Takashi Ouchi ${ }^{1}$
}

\begin{abstract}
Background: Remimazolam is a newly developed benzodiazepine with more rapid onset and offset of sedation effects than midazolam. We report elderly patients in whom a small dose of remimazolam was successfully used for general anesthesia.

Case presentation: Two elderly women (patients 1 and 2, aged 95 and 103 years, respectively) underwent hip fracture surgery under general anesthesia guided by bispectral index (BIS). Anesthesia was induced with 1.2 and 1.0 $\mathrm{mg} / \mathrm{kg} / \mathrm{h}$ and maintained with 0.2 and $0.1 \mathrm{mg} / \mathrm{kg} / \mathrm{h}$ remimazolam, combined with fentanyl and remifentanil in patients 1 and 2, respectively. Their hemodynamics were stable with a small dose of vasopressor, and they awoke soon after the discontinuation of remimazolam without flumazenil reversal. Their postoperative courses were uneventful without any complications. Conversely, the remimazolam dose required to achieve adequate sedation were much lower than expected.

Conclusion: Remimazolam could be useful in general anesthesia, particularly for super-elderly patients. However, the appropriate dose for induction and maintenance of anesthesia should be carefully considered based on BIS or vital signs.
\end{abstract}

Keywords: Remimazolam, Super-elderly, Bispectral index

\section{Background}

Remimazolam, a new ultra-short-acting benzodiazepine, was approved for general anesthesia on January 23, 2020, in Japan for the first time in the world [1-3]. The effect of this agent rapidly appears and disappears compared with midazolam $[4,5]$. Moreover, similar to other benzodiazepines, remimazolam agent can cause minimal cardiorespiratory depression [6-8]. These characteristics imply that remimazolam is particularly effective for short-term anesthesia in patients with poor performance status, including super-elderly patients. However, there is little real-world information on remimazolam under general anesthesia.

\footnotetext{
* Correspondence: junko.t@keio.jp

${ }^{1}$ Department of Anesthesiology, Tokyo Dental College Ichikawa General

Hospital, 5-11-13 Sugano, Ichikawa City, Chiba 272-8513, Japan

Full list of author information is available at the end of the article
}

This report presents two cases of super-elderly women aged $\geq 95$ years who underwent hip fracture surgery under general anesthesia using remimazolam guided by the bispectral index (BIS) or monitoring of the vital signs.

\section{Case presentation \\ Case 1}

A 95-year-old woman $(135 \mathrm{~cm}$ and $40 \mathrm{~kg})$ presented with right intertrochanteric femoral fracture requiring surgery. She had a history of hypertension, dyslipidemia, and dementia, which were controlled by amlodipine and olmesartan, pravastatin, and donepezil, respectively. Physical examination findings were normal, and laboratory examination revealed mild anemia with hemoglobin $(\mathrm{Hb})$ level of $11.8 \mathrm{~g} / \mathrm{dL}$ and coagulopathy of prothrombin time $[\mathrm{PT}]$ of $58 \%$ (PT-international normalized ratio,
Springer Open

(c) The Author(s). 2021 Open Access This article is licensed under a Creative Commons Attribution 4.0 International License, which permits use, sharing, adaptation, distribution and reproduction in any medium or format, as long as you give appropriate credit to the original author(s) and the source, provide a link to the Creative Commons licence, and indicate if changes were made. The images or other third party material in this article are included in the article's Creative Commons licence, unless indicated otherwise in a credit line to the material. If material is not included in the article's Creative Commons licence and your intended use is not permitted by statutory regulation or exceeds the permitted use, you will need to obtain permission directly from the copyright holder. To view a copy of this licence, visit http://creativecommons.org/licenses/by/4.0/. 
1.3) probably owing to advanced age. Transthoracic echocardiography, electrocardiography, and respiratory function test revealed no abnormalities.

Anesthetic chart is shown in Fig. 1. In the operating room, the patient's vital signs were as follows: blood pressure (BP), 136/70 $\mathrm{mmHg}$; heart rate, $88 \mathrm{bpm}$; and oxygen saturation on room air, $92 \%$. BIS value was $>90$ before inducing anesthesia. General anesthesia was induced by intravenous administration of fentanyl $(50 \mu \mathrm{g})$, remifentanil $(0.15 \mu \mathrm{g} / \mathrm{kg} / \mathrm{min})$, and remimazolam $(1.2$ $\mathrm{mg} / \mathrm{kg} / \mathrm{h}$ ). She lost consciousness $4 \mathrm{~min}$ after initiation, and subsequently tracheal intubation was performed after administration of rocuronium $(40 \mathrm{mg})$. After the operation was started, the remimazolam dose was adjusted so that the BIS value became 40-60 concomitantly with $0.125 \mu \mathrm{g} / \mathrm{kg} / \mathrm{min}$ of remifentanil infusion. Eventually, remimazolam dose was decreased to $0.2 \mathrm{mg} / \mathrm{kg} / \mathrm{h}$ although BIS value remained $<40$. The operation was uneventfully completed with a small amount of blood loss in approximately $90 \mathrm{~min}$. During the operation, she remained hemodynamically stable with administration of ephedrine $4 \mathrm{mg}$ bolus and phenylephrine $50 \mu \mathrm{g}$ bolus only twice to maintain systolic BP at $>90$ $\mathrm{mmHg}$, without the need of continuous administration of vasopressor agents. At the time of suture, $50 \mu \mathrm{g}$ of fentanyl was administered, the remifentanil dose was decreased to $0.075 \mu \mathrm{g} / \mathrm{kg} / \mathrm{min}$, and remimazolam was maintained at $0.2 \mathrm{mg} / \mathrm{kg} / \mathrm{h}$. After completion of the operation, administration of these agents was discontinued and $200 \mathrm{mg}$ of sugammadex was administered. Although $2.0 \mathrm{mg}$ of remimazolam was administered as a bolus owing to the BIS value of $>70$ during postural change for radiographic examination, $10 \mathrm{~min}$ after the bolus administration, she recovered consciousness without flumazenil and received extubation. Any consciousness problem, including resedation or postoperative cognitive function disorder, was not observed during the postoperative course. After rehabilitation, she was discharged on postoperative day 15.

\section{Case 2}

A 103-year-old woman (approximately $150 \mathrm{~cm}$ and 40 $\mathrm{kg}$ ) presented with right intertrochanteric femoral fracture requiring surgery. She had a history of chronic kidney disease (CKD) due to hypertension, which was treated with amlodipine, furosemide, and valsartan. Moreover, she took ticlopidine for the prevention of cerebral infarction. Physical examination results were normal, and laboratory examination revealed stage $3 \mathrm{~b}$ CKD with serum creatinine level of $1.18 \mathrm{mg} / \mathrm{dL}$ (estimated glomerular filtration rate, $31.6 \mathrm{~mL} / \mathrm{min} / 1.73 \mathrm{~m}^{2}$ ) and mild anemia with $\mathrm{Hb}$ level of $10.0 \mathrm{~g} / \mathrm{dL}$. Transthoracic echocardiography and electrocardiography revealed no abnormalities.

Anesthetic chart is shown in Fig. 2. In the operating room, she was hemodynamically stable with vital signs as follows: BP, $157 / 58 \mathrm{mmHg}$, heart rate, $84 \mathrm{bpm}$; and oxygen saturation on room air, 94\%. BIS value was $>90$ before anesthesia. General anesthesia was induced by intravenous administration of fentanyl $(100 \mu \mathrm{g})$, remifentanil $(0.3 \mu \mathrm{g} / \mathrm{kg} / \mathrm{min})$, and remimazolam $(1 \mathrm{mg} / \mathrm{kg} / \mathrm{h})$. She lost consciousness $3 \mathrm{~min}$ after initiation, and subsequently tracheal intubation was performed after administration of rocuronium $(40 \mathrm{mg})$. After the operation was

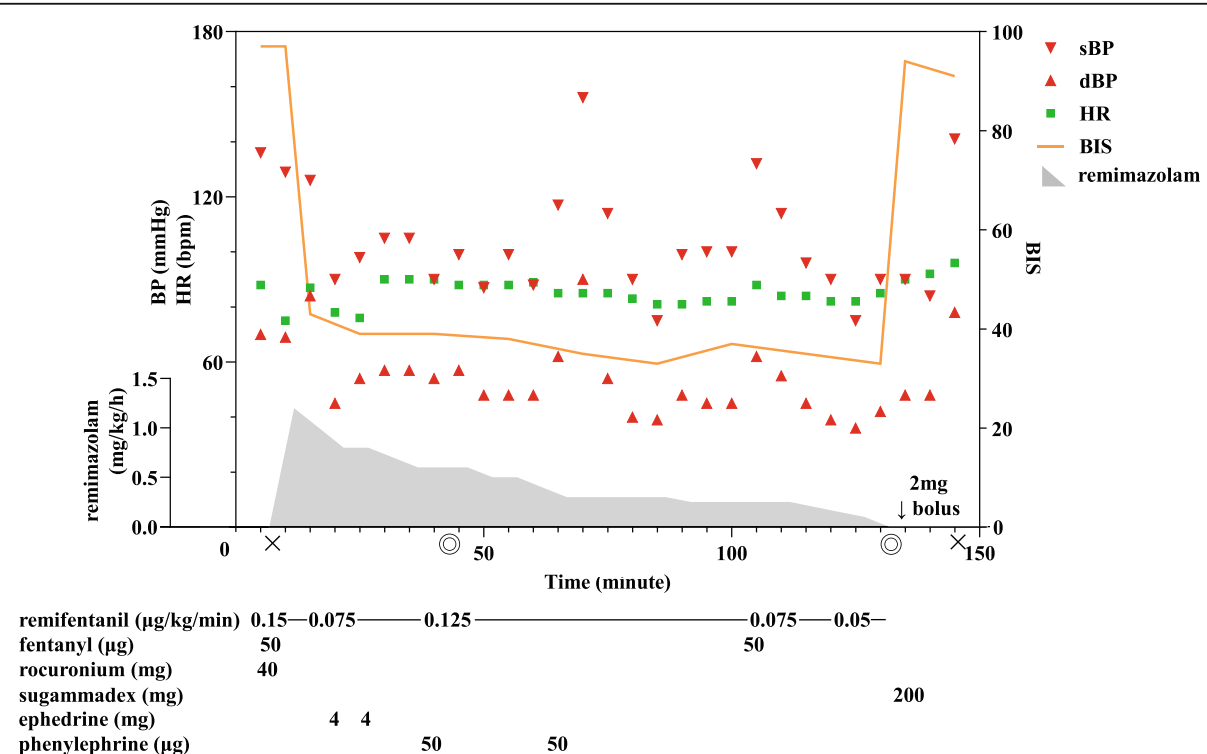

Fig. 1 Anesthetic chart for case 1. Double circles and crosses represent the start/end of surgery and start/end of anesthesia, respectively. sBP, systolic blood pressure; dBP, diastolic blood pressure; HR, heart rate; BIS, bispectral index 


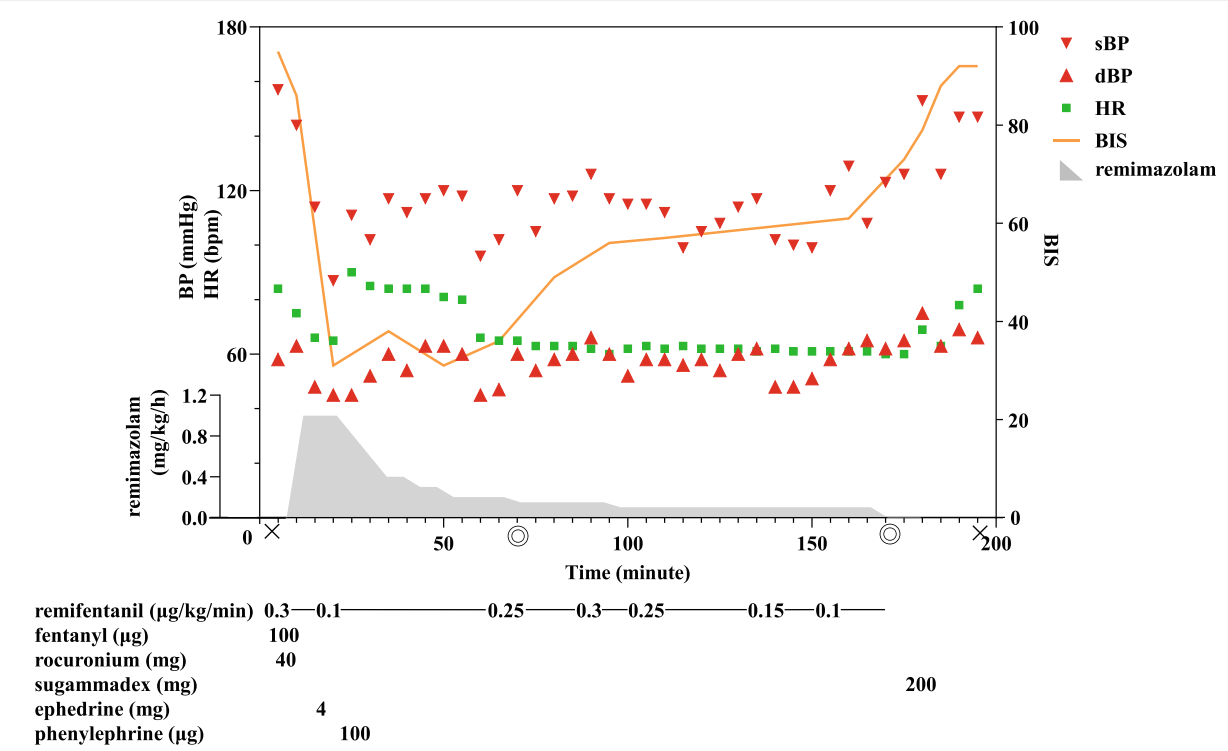

Fig. 2 Anesthetic chart for case 2. Double circles and crosses represent the start/end of surgery and start/end of anesthesia, respectively. sBP, systolic blood pressure; dBP, diastolic blood pressure; HR, heart rate; BIS, bispectral index

started, the remimazolam dose was adjusted so that the BIS value became $40-60$ concomitantly with $0.1-0.3 \mu \mathrm{g} /$ $\mathrm{kg} / \mathrm{min}$ of remifentanil infusion. Eventually, the remimazolam dose was decreased to $0.1 \mathrm{mg} / \mathrm{kg} / \mathrm{h}$ with BIS value of approximately 50-60. The operation was uneventfully completed with a small amount of blood loss in approximately $100 \mathrm{~min}$. During the operation, she remained hemodynamically stable without administration of any vasopressor agents, except when general anesthesia was induced. After completion of the operation, administration of remifentanil and remimazolam was discontinued and $200 \mathrm{mg}$ of sugammadex was administered; $8 \mathrm{~min}$ after discontinuation of these agents, she recovered consciousness without flumazenil and received extubation. Any consciousness problem, including resedation or postoperative cognitive function disorder, was not observed during the postoperative course. After rehabilitation, she was discharged on postoperative day 29.

\section{Discussion}

The two patients aged $\geq 95$ years had satisfactory intraoperative management with remimazolam. Their hemodynamics were stable during operation, and prompt emergence from anesthesia was achieved. Conversely, it is remarkable that the required maintenance dose could be much lower than the expected dose $[2,3]$.

Benzodiazepine is widely known to have less circulation effect than other sedatives for general anesthesia, such as propofol or inhalation anesthetics [6, 7]. The effect could be the same for a newly developed benzodiazepine, remimazolam [8]. In the present cases, although our patients were very elderly and relatively hypovolemic, vasopressor agents were almost not required to maintain hemodynamics. This property of remimazolam is beneficial for patients with unstable hemodynamics, including super-elderly patients or patients with hemorrhagic or hypovolemic hypotension, severe cardiac disease, or arteriosclerotic disease. The duration of action of remimazolam is much shorter than that of midazolam, and it appears that remimazolam could be administered without paying too much attention to prolonged effect or resedation after administration of the reversal agent [4]. The extremely short duration of action of remimazolam seems beneficial for a relatively short-term operation. In fact, we could achieve prompt emergence from general anesthesia without the use of reversal agents.

The required dose of remimazolam to maintain BIS value of 40-60 was much lower than those required in previous randomized controlled trials (RCTs) (bolus dose, 6.0 or $12.0 \mathrm{mg} / \mathrm{kg} / \mathrm{h}$; mean optimal maintenance dose, $0.99 \mathrm{mg} / \mathrm{kg} / \mathrm{h}$ for American Society of Anesthesiologists [ASA] class I and II patients and $0.56 \mathrm{mg} / \mathrm{kg} / \mathrm{h}$ for ASA class III patients) [2, 3]. There is room for examination using appropriate doses, particularly for superelderly patients. In the present cases, because both patients were very elderly with possible mild hypovolemia, we induced anesthesia with an extremely low dose (bolus dose, $1.2 \mathrm{mg} / \mathrm{kg} / \mathrm{h}$ for case 1 and $1.0 \mathrm{mg} / \mathrm{kg} / \mathrm{h}$ for case 2) [3]. However, they lost consciousness only a few minutes after the start of administration. The maintenance doses to achieve BIS values of 40-60 were $0.2 \mathrm{mg} /$ $\mathrm{kg} / \mathrm{h}$ for case 1 and $0.1 \mathrm{mg} / \mathrm{kg} / \mathrm{h}$ for case 2 , which were much lower than those for ASA class III patients in the 
previous RCT [3]. Furthermore, in case 1, the maintenance dose of remimazolam was likely to be excessive from the facts that the BIS value was $<40$ and electroencephalography often showed burst-suppression patterns [9]. We were hesitant to significantly reduce the dose compared with RCTs, which could be inappropriate. These findings showed that there could be considerable differences in the appropriate dose of remimazolam among individual characteristics, including age and circulatory state as well as other benzodiazepine class anesthetics $[10,11]$. In fact, the Japanese phase 2 study shows that both maintenance dose and time to extubation varied widely, and $8 \%$ of patients (29/362 cases) need $>30$ min to become conscious [2]. The appropriate dose appears to greatly depend on multiple factors, such as age, genetic sensitivity, circulating blood volume, cardiac and liver function, and dose of concomitant opioid. The doses required for our patients were dramatically low; this was primarily owing to their hypersensitivity to sedatives due to their old age, low circulating blood volume, and sufficient dose of remifentanil administration.

It is doubtful that an excessive amount of remimazolam is safe even if the offset period is very short and the reversal agent is available. Deep intraoperative anesthesia is associated with not only postoperative cognitive function disorder but also mortality [12-14]. Most recently, the coronavirus disease 2019 pandemic has resulted in the shortage of sedatives worldwide, due to which clinicians need to ascertain appropriate doses of it $[15,16]$.

We encountered cases of two patients aged $\geq 95$ years who were administered general anesthesia using remimazolam. Remimazolam is a promising agent, particularly for the super-elderly or critically ill patients under general anesthesia to achieve stable hemodynamics. However, dose adjustment needs careful attention. The optimal dose for elderly patients could be much lower as expected. Future research is warranted to clarify the optimal dose for super-elderly patients.

\section{Abbreviations}

ASA: American Society of Anesthesiologists; BIS: Bispectral index; CKD: Chronic kidney disease; PT: Prothrombin time; RCT: Randomized controlled trials

\section{Acknowledgements}

We thank Enago (www.enago.jp) for the English language review.

\section{Authors' contributions}

JN wrote the initial draft of the manuscript. JN, TO1, and RY were in charge of inducing and maintaining anesthesia and contributed to data collection. $\mathrm{YI}$ and TO2 critically revised the manuscript. All authors read and approved the final manuscript.

\section{Funding}

The authors declare no funding for this report.

Availability of data and materials

Not applicable

\section{Declarations}

Ethics approval and consent to participate

Not applicable

\section{Consent for publication}

Written informed consent for patient information to be published was provided by the patients.

\section{Competing interests}

The authors have declared that no conflict of interests exists.

\section{Author details}

'Department of Anesthesiology, Tokyo Dental College Ichikawa General Hospital, 5-11-13 Sugano, Ichikawa City, Chiba 272-8513, Japan. ${ }^{2}$ Department of Anesthesiology, Itakura Hospital, Chiba, Japan.

Received: 1 July 2021 Revised: 3 September 2021

Accepted: 6 September 2021 Published online: 15 September 2021

\section{References}

1. Masui K. Remimazolam besilate, a benzodiazepine, has been approved for general anesthesia!! J Anesth. 2020;34(4):479-82. https://doi.org/10.1007/ s00540-020-02755-1.

2. Doi M, Morita K, Takeda J, Sakamoto A, Yamakage M, Suzuki T. Efficacy and safety of remimazolam versus propofol for general anesthesia: a multicenter, single-blind, randomized, parallel-group, phase IIb/III trial. J Anesth. 2020; 34(4):543-53. https://doi.org/10.1007/s00540-020-02788-6.

3. Doi M, Hirata N, Suzuki T, Morisaki H, Morimatsu H, Sakamoto A. Safety and efficacy of remimazolam in induction and maintenance of general anesthesia in high-risk surgical patients (ASA Class III): results of a multicenter, randomized, double-blind, parallel-group comparative trial. J Anesth. 2020;34(4):491-501. https://doi.org/10.1007/s00540-020-02776-w.

4. Antonik L, Goldwater D, Kilpatrick G, et al. A placebo- and midazolamcontrolled phase I single ascending-dose study evaluating the safety, pharmacokinetics, and pharmacodynamics of remimazolam (CNS 7056): part I. Safety, efficacy, and basic pharmacokinetics. Anesth Analg. 2012;115(2): 274-83. https://doi.org/10.1213/ANE.0b013e31823f0c28.

5. Lohmer LL, Schippers F, Petersen KU, Stoehr T, Schmith VD. Time-to-event modeling for remimazolam for the indication of induction and maintenance of general anesthesia. J Clin Pharmacol. 2020;60(4):505-14. https://doi.org/10.1002/jcph.1552.

6. Frölich MA, Arabshahi A, Katholi C, Prasain J, Barnes S. Hemodynamic characteristics of midazolam, propofol, and dexmedetomidine in healthy volunteers. J Clin Anesth. 2011;23(3):218-23. https://doi.org/10.1016/j.jclina ne.2010.09.006.

7. Han D, Liu YG, Pan SD, Luo Y, Li J, Ou-Yang C. Comparison of sufentanilmidazolam and sevoflurane for anesthesia induction in children undergoing cardiac surgery by real-time hemodynamic and cardiac efficiency monitoring: a prospective randomized study. Heart Surg Forum. 2019;22(1): 38-44. https://doi.org/10.1532/hsf.2037.

8. Sato S, Doi, M, Morita, K,etal., editor Remimazolam a new ultra-short actinganesthetic shows similar efficacy and superior hemodynamic stability vs propofol in general. Chicago, USA: Poster Session Presented at the ANESTHESOLOGY 2015 Annual Meeting. Abstract Number: A5018;2015.

9. Bruhn J, Bouillon TW, Shafer SL, et al. Bispectral index (BIS) and burst suppression: revealing a part of the BIS algorithm. J Clin Monit Comput. 2000;16(8):593-6. https://doi.org/10.1023/A:1012216600170.

10. Yamashita K, Terao Y, Inadomi C, Takada M, Fukusaki M, Sumikawa K. Agedependent relationship between bispectral index and sedation level. J Clin Anesth. 2008;20(7):492-5. https://doi.org/10.1016/j.jclinane.2008.05.004.

11. Platten HP, Schweizer E, Dilger K, Mikus G, Klotz U. Pharmacokinetics and the pharmacodynamic action of midazolam in young and elderly patients undergoing tooth extraction. Clin Pharmacol Ther. 1998;63(5):552-60. https://doi.org/10.1016/S0009-9236(98)90106-0.

12. Chan MT, Cheng BC, Lee TM, Gin T, CODA Trial Group. BIS-guided anesthesia decreases postoperative delirium and cognitive decline. J Neurosurg Anesthesiol. 2013;25(1):33-42. https://doi.org/10.1097/ANA.0b013 e3182712fba.

13. Lindholm ML, Traff S, Granath F, et al. Mortality within 2 years after surgery in relation to low intraoperative bispectral index values and preexisting 
malignant disease. Anesth Analg. 2009;108(2):508-12. https://doi.org/1 0.1213/ane.0b013e31818f603c.

14. Leslie K, Myles PS, Forbes A, Chan MTV. The effect of bispectral index monitoring on long-term survival in the B-aware trial. Anesth Analg. 2010; 110(3):816-22. https://doi.org/10.1213/ANE.0b013e3181c3bfb2.

15. Ammar MA, Sacha GL, Welch SC, Bass SN, Kane-Gill SL, Duggal A, et al. Sedation, analgesia, and paralysis in COVID-19 patients in the setting of drug shortages. J Intensive Care Med. 2021;36(2):157-74. https://doi.org/1 $0.1177 / 0885066620951426$.

16. Food and Drug Administration. https://www.fda.gov/drugs/drug-safety-a nd-availability/drug-shortages. Accessed 23 June 2021

\section{Publisher's Note}

Springer Nature remains neutral with regard to jurisdictional claims in published maps and institutional affiliations.

\section{Submit your manuscript to a SpringerOpen ${ }^{\circ}$ journal and benefit from:}

- Convenient online submission

- Rigorous peer review

- Open access: articles freely available online

High visibility within the field

- Retaining the copyright to your article

Submit your next manuscript at $\boldsymbol{\wedge}$ springeropen.com 